\title{
APPLICATION FOR STUDENT ATTENDANCE BASED ON FACE RECOGNITION
}

\author{
Aleksa Ćuk \\ Branivoj Miljković, \\ Miloš Todorović, \\ Aleksandar Ivanović, \\ Miodrag Živković
}

Singidunum University,

Belgrade, Serbia
Correspondence:

Aleksa Ćuk

e-mail:

aleksa.cuk.15@singimail.rs

\begin{abstract}
:
Facial recognition represents a system for automatic recognition or identification of persons based on digital pictures of their face. There are multiple algorithms that the recognition software uses but it all comes down to comparison of input picture, that is the comparison of the biometric face features with the selected face pictures in the database. Facial recognition is mostly used in systems that are connected with safety and usually in two ways: recognition and verification. When it comes to recognizing the face parameters are compared with other faces in the database. For the verification, the face parameters of the person that is using another way of verification in the given moment, are compared with the information of that person in the database. This is done for the sake of making sure that the person who is requesting access is actually a person with allowed access. Even though facial recognition is less precise compared to fingerprint scanning or iris scanning, it is very well accepted because of its non-physical and non-invasive way of use.
\end{abstract}

Keywords:

face recognition, authentication, deep learning, PCA algorithm.

\section{INTRODUCTION}

The problems of conventional evidencing are inconsistency and the time it requires. It is efficient and consistent for keeping track of attendance of students on classes. The main characteristic of the program is recognizing and evidencing the attendance. This way the process is completely automatized since the presence is automatically evidenced and the time for manual noting of attendance is eliminated.

In the first step, it is necessary to make sure that the student is registered in the database. In the case that they aren't registered, the student should contact the administrator for the creation of their account.

Necessary hardware support for our project is a camera on which our registration is done, that is on which is the software installed. The purpose of the camera is to do proper registration of the user. There are no prerequisites for the camera). From the software side we have the communication between our main application and the database, which contains images of the people who are registered. After that, the user sees on 
the screen a message which tells him to remove glasses (if there are any) and to face the camera face front for the reason of precise recognition.

After the recognition, the user gets a message from the device about the successful registration.

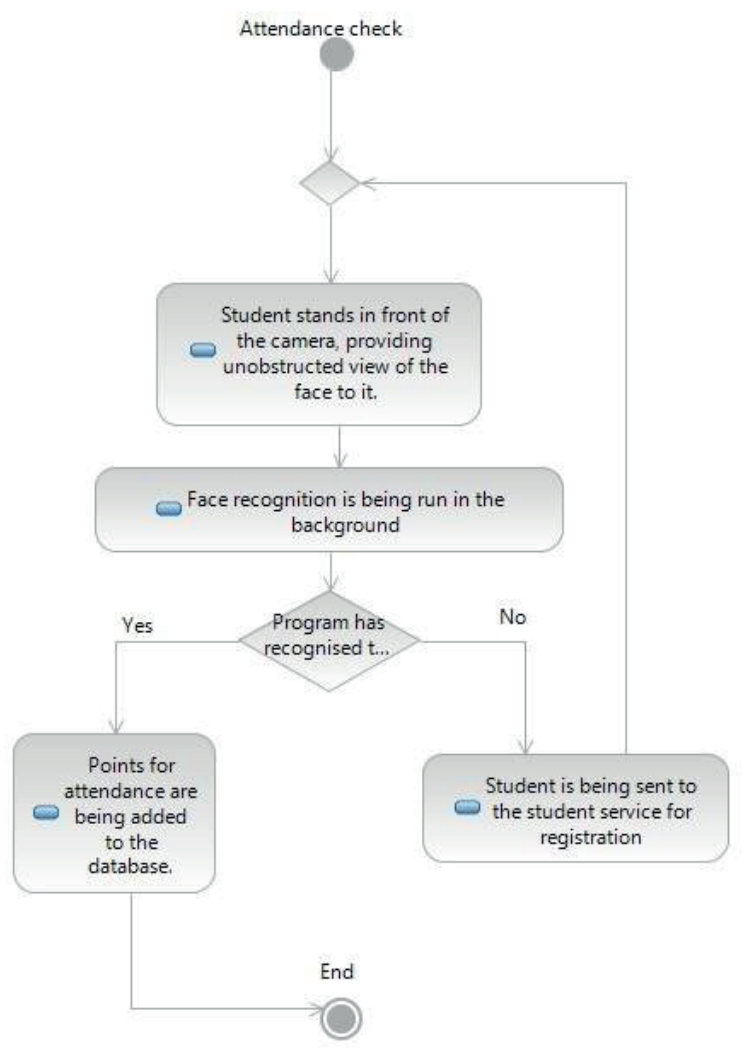

Fig. 1. Structure of our program

\section{BACKGROUND}

Authentication is a significant issue in system control in computer based communication. Human face recognition is an important branch of biometric verification and has been widely used in many applications, such as video monitor system, human-computer interaction, and door control system and network security. This paper describes a method for Student's Attendance System which will integrate with the face recognition technology using Personal Component Analysis (PCA) algorithm[1]. The system will record the attendance of the students in class room environment automatically and it will provide the facilities to the faculty to access the information of the students easily by maintaining a $\log$ for clock-in and clock-out time.
Over the past decade face detection and recognition have transcended from esoteric to popular areas of research in computer vision and one of the better and successful applications of image analysis and algorithm based understanding. Facial recognition generally involves two stages: Face Detection where a photo is searched to find a face, then the image is processed to crop and extract the person's face for easier recognition. Face Recognition where that detected and processed face is compared to a database of known faces, to decide who that person is. Since 2002, face detection can be performed fairly easily and reliably with Intel's open source framework called OpenCV. This framework has an inbuilt Face Detector that works in roughly $90-95 \%$ of clear photos of a person looking forward at the camera. However, detecting a person's face when that person is viewed from an angle is usually harder, sometimes requiring $3 \mathrm{D}$ Head Pose Estimation. Also, lack of proper brightness of an image can greatly increase the difficulty of detecting a face, or increased contrast in shadows on the face, or maybe the picture is blurry, or the person is wearing glasses, etc. Face recognition however is much less reliable than face detection, with an accuracy of $30-70 \%$ in general[2]. The Eigenface technique is considered the simplest method of accurate face recognition, but many other (much more complicated) methods or combinations of multiple methods are slightly more accurate.

\section{FACIAL RECOGNITION}

Facial recognition is a biometric software application capable of uniquely identifying or verifying a person by comparing and analyzing patterns based on the person's facial contours[3].

Facial recognition is mostly used for security purposes, though there is increasing interest in other areas of use. There are different facial recognition techniques in use, such as the generalized matching face detection method and the adaptive regional blend matching method. Most facial recognition systems function based on the different nodal points on a human face. The values measured against the variable associated with points of a person's face help in uniquely identifying or verifying the person.

Facial recognition techniques are quickly evolving with new approaches such as 3-D modeling, helping to overcome issues with existing techniques. 


\section{DEEP LEARNING}

Deep learning is a part of machine learning which is convenient for solving problems from artificial intelligence, where algorithms are inspired by human brain, do calculation and learn from huge number of data. We refer to 'deep learning' because the neural networks have various layers that enable learning. Similarly to human way of learning, deep learning algorithm is learning from experience [4]. Methods from deep learning find their application in challenging fields where the size of data is exceptionally big: computer vision, processing of speech.

These are some examples of deep learning and list will continue to grow in future:

\section{- Facial recognition}

Face recognition is starting to be a normal thing in our lives, we start seeing it in every aspect of our daily routine. Face recognition can be used for unlocking phones or using some filters for apps like Instagram or Snapchat.

\section{- Virtual assistants}

In the world of Alexa, Siri and other, the virtual assistants of online service providers use deep learning to help understand your speech and the language humans use when they interact with them.

\section{- Translations}

In a similar way, deep learning algorithms can automatically translate between languages. This can be powerful for travelers, business people and those in government.

\section{- Autonomous cars}

Also known as a self-driving car is already being developed by the Tesla, BMW and Mercedes, Google also developing his own automated technology. Self-driving cars is still at testing stage, but partially automated technology has been around for the last few years, a car can park themselves, or follow a car in front of them or road lines.

\section{- Medicine and pharmaceuticals}

Deep learning can be use in various fields of medicine, from disease and tumor diagnoses to personalized medicines created specifically for an individual's genome.

\section{APP DESCRIPTION}

Idea

Facial recognition is a really a series of several related problems. First of all, it takes a look at a picture and finds all the faces in it. Secondly, focus on each face and be able to understand that even if a face is turned in a weird direction or in bad lighting, it is still the same person. Third it is able to pick out unique features of the face so that it can tell them apart from other people (Fig.1.).

Finally it will compare unique features of that face to all the people you already know to determine the person's name. This is all done since computers aren't able of high-level generalization. So we solve this by basically leading it though the steps and separately passing the results of each step.

\section{Finding Faces}

For the finding of faces in a picture HOG (Histogram of Oriented Gradients) is used (Fig.3.). That is, the first step in the process is that the picture is turned to grayscale. The point of this is to figure out how dark are the pixels compared to other pixels surrounding it and arrow is drawn that will show in which way the image is getting darker. This process is repeated for every pixel in the image, until each one is replaced by with an arrow. This actually gives us the gradient for the picture. But since saving the gradient for every single pixel gives us way too much detail, we'll break up the image in to a square of $16 \times 16$ pixels each and each square we will count up how many gradients point in each major direction. From this we get a greyscale picture that in some way represents a HOG pattern.

As for the percentage of the successful recognition, since in this phase we only implement the pictures in the code, the percentage is over $98 \%$. This is for the reason that currently we only use one sample picture of the person that should use the database and one picture of a person that is not desired, for the sake of comparison. This other person can also be a desired person for the recognition while the other one is used for the comparison. There is also a simple flaw, that if a unregistered person has very similar face features and hairstyle compared to a person who's picture is already in the code, the person might get up to $86 \%$ successful recognition, but inconsistently (with spikes from $54 \%$ up to $86 \%$ and changes in the milliseconds). 


\section{Posing and Projecting Faces}

After we isolated the faces in our image, we have to deal with a problem with direction of the face. The basic idea is, we will come up with 68 points that exist on every face, top of the chin, outside edge of each eye, the inner edge of each eyebrow. We will obtain this 68 point using the face landmark estimation. After that we will train machine learning [5]. After that we will train machine learning algorithm to be able to fine these 68 specific points on any face:

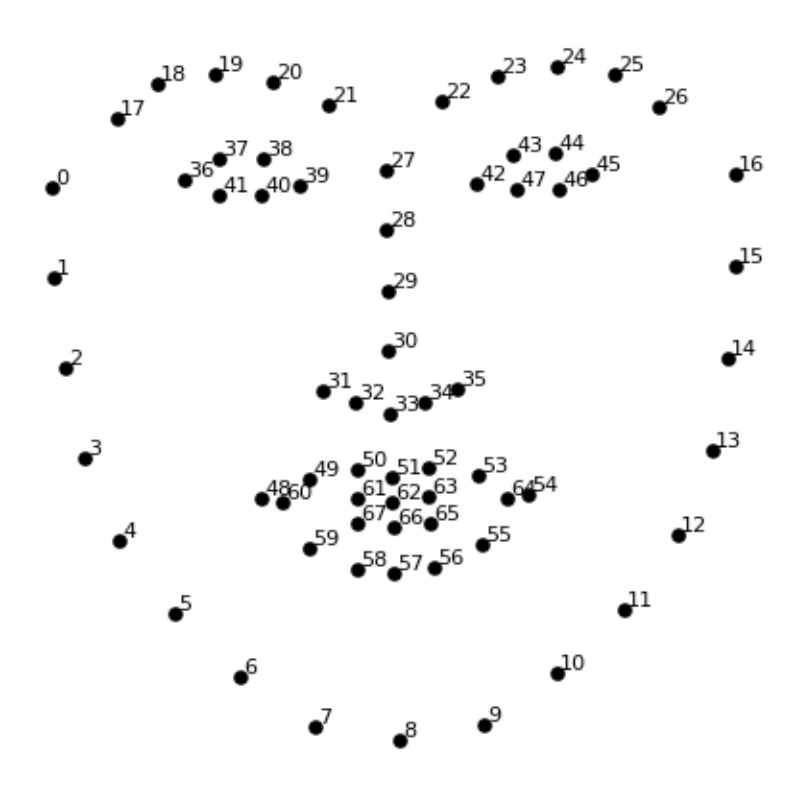

Fig. 2. Example of 68 face landmark

\section{Encoding faces}

Now we approach the problem of telling faces apart. This can be simply solved if we directly compare the face we find in step two with all the pictures we have of people in our database. For this to be done we would need to extract a few basic measurements from each face. This basic measurements are the 68 point from the face we got from the face landmark estimation (Fig.2.). From here we will turn these 68 point to the 128 measurements that will be compared to the 128 measurements in our picture in the database(Fig.4.).

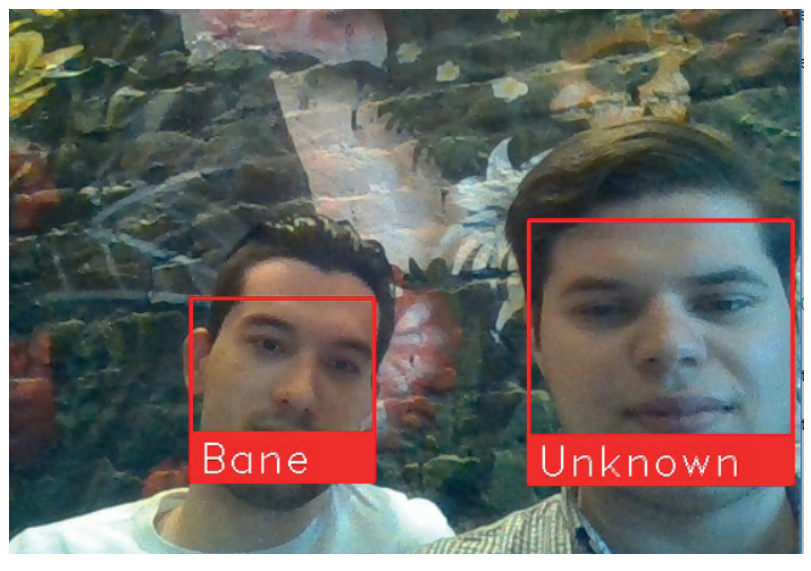

Fig. 3. Example of face recognition of two persons: one in database, one that is not
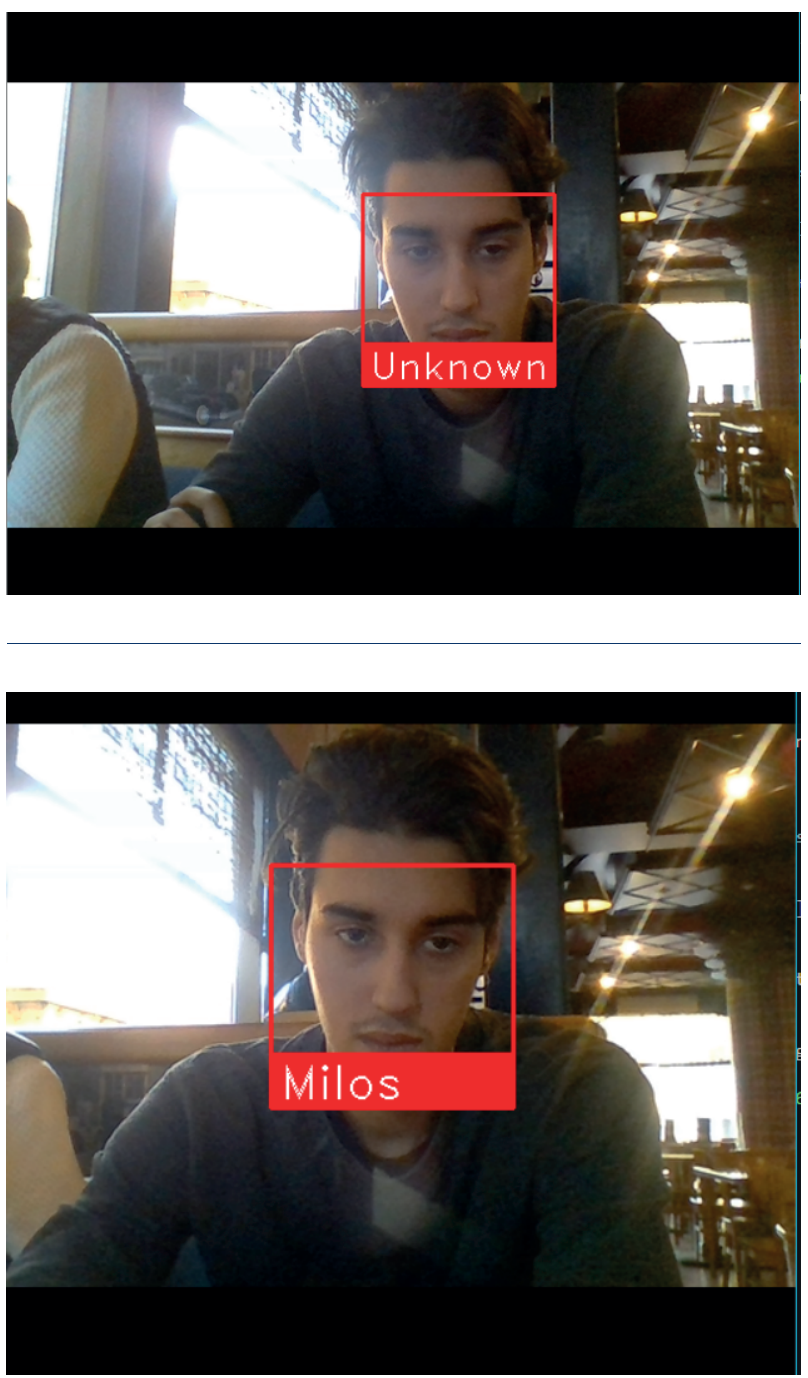

Fig.4. Example of a person before and after being registered 


\section{IMPLEMENTATION}

\section{Python}

Python is a high level general-purpose programming language released 1991. It enables programming on both large and small scale. It features a dynamic type and automatic memory management. Python supports objectoriented, imperative, functional and procedural. It has a large supported standard library.

\section{OpenCV}

OpenCV has the advantage of being a multi-platform framework. It has so many capabilities it can seem overwhelming at first. A good understanding of how these methods work is the key to getting good results when using OpenCV. Fortunately, only a select few need to be known beforehand to get started. OpenCV's functionality that will be used for facial recognition is contained within several modules.

\section{APPLICATION IN THE REAL WORLD}

Since the usage of intelligent software is more and more present, facial recognition is also becoming a part of our day to day routine and there's no reason why it shouldn't be used for the automatization of taking evidence of attendance [6]. This represents our small contribution to modernizing the education system.

Examples of facial recognition in real life:

- Prevent retail crime - It lowers crime rates, by identifying know criminals when they enter retail establishments. Their photographs are kept in a database of criminals and the management is notified when a notorious person enters their shop.

- Unlock phones - This is where facial recognition has found most application up to date. Even though to some sense it is less consistent compared to fingerprint scanning, it is practically impossible to be surpassed by an undesired individual. For this reason, its ease of use and convenience, it is clear why its so used today in this field.

- Smarter advertising - It is somewhat used in advertising as well. The idea is to display more appealing adds to people based on their age and gender. Its usefulness is still being tested in this category.
- Find missing persons - One of the humane uses of facial recognition is to help fight against human trafficking. By ensuring that the missing persons are entered in the databases, we notify the cameras at public places (airports, train stations etc.) to be on alert for them and to notify the authorities.

- Help the blind - One of the groundbreaking implementations is helping blinding people in communicating in social situations. The app will notify the blind person with vibrations when the interlocutor is smiling.

- Protect law enforcement - Police is already using facial recognition in their daily routine. By being able to identify people from a distance, police officer can know how to approach situations more appropriately.

- Aid forensic investigations - Forensics also getting a helping hand with this technology by making the identification of people possible even though they might not be able to communicate in the given moment. And as well as tracking them on security footage.

- Identify people in social media - Identification on social media can make recognizing people much easier. May it be in the sense of making a suggestion for a tag on a picture or linking multiple accounts of the same person.

- Diagnose diseases - We can also identify certain diseases which manifest their symptoms through a person's face. An example is a rare disease DiGeorge syndrome. Facial recognition helped diagnose the disease in $96 \%$ of cases.

- Recognize VIPs at sporting events - It can also help with making the experience of people of interest more pleasant. An example would be VIPs at sporting events. By recognizing their picture on their seasonal pass, it can offer them better seats, exclusive parties and merchandise.

- Protect schools for threats - Facial recognition also offers aid at keeping public schools safer. By maintain a database of people who are not wanted in a school (expelled students, drug dealers etc.) the school security can be notified immediately when those people enter the schools property.

- Casinos - It can prevent card counters and known cheats to enter casinos if they have been registered as unwanted in their database. 
- Facilitate secure transactions - It can be used as the only means in a transaction. By connecting a person's bank account to their information and an identification picture, we can completely eliminate credit cards and provide our face as credentials in a transaction.

- Control access to sensitive areas - It can be also used as another safety step when controlling dangerous facilities. That is beside a id card a face can would be required as well before entering laboratories, military bases, bank vaults etc.

\section{CONCLUSIONS AND FUTURE WORK}

The goal of our project is to make keeping track of attendance at events easier. There are many ways to evident people at events, for example using personalized cards, taking signatures at the beginning of an event, but we found that facial recognition is more practical than any other conventional way. We found that using facial recognition is quite challenging and we tackled many problems throughout the development. In the beginning there was a decision to be made, do we use a face recognition algorithm or do we use face encodings. We found that the latter one is more suitable for our application. Another decision that we made is to implement the pictures directly into our program instead of using a database, but only for our testing purposes. |Both the face recognition algorithms and a database can be easily implemented in the future, as the number of the users grows.
We left a lot of space for flexibility and testing to be done on our program. That includes testing its consistency as the number of users grows, as well as defining its specific purpose whether it is used for keeping attendance of students on a class, evidencing visitants on a meeting or seminar, or just simply keeping track of reservations in a restaurant. Depending on its specific purpose, it can be modified with simple implementations to fulfil the user's needs.

\section{REFERENCES}

[1] N. Kar, M. K. Debbarma, A. Saha and D. R. Pal, "Study of implementing automated attendance system using face recognition technique," International Journal of computer and communication engineering, vol. 1, no. 2, 2012.

[2] S. Emami, V. P. Suciu, "Facial recognition using OpenCV," Journal of Mobile, Embedded and Distributed Systems, vol. 4, no. 12012.

[3] R. Shanmugamani, A.G. Abdul Rahman, S. M. Moore, N. Koganti, "Deep Learning for Computer Vision: Expert techniques to train advanced neural networks using TensorFlow and Keras," Packt Publishing, Birmingham, 2018.

[4] F. Chollet, "Deep Learning with Python," Manning Publications, Co., Greenwich, CT, US, 2017.

[5] A. Rosebrock, "Deep Learning for Computer Vision with Python: Starter Bundle," PyImageSearch, 2017.

[6] M. Milosavljević, "Veštačka inteligencija," Univerzitet Singidunum, Beograd, 2015. 